\title{
1-S01-2 Symposium1
}

\section{Neuronal Signaling in Islet Development and Function}

\section{Rejii Kuruvilla}

\author{
Department of Biology, Johns Hopkins University
}

Pancreatic islets, the functional units in regulating blood glucose levels, are richly innervated by sympathetic nerves. Employing genetic mouse models and neuron-islet co-cultures, we discovered that sympathetic innervation is essential for organizing islet structure during development (Borden et al., Cell Rep. 2013). Further, we demonstrated that this inductive interaction at early stages is critical for mature islet function in regulating glucose metabolism, suggesting that developmental perturbations in sympathetic innervation might underlie metabolic dysfunction in humans. We are building on these findings to identify the molecular pathways by which sympathetic nerves instruct islet organization and the acquisition of functional maturity. To identify how sympathetic nerves influence islets, we recently asked whether neuronal activity is required for islet formation and function. Sympathetic nerves innervating the pancreas secrete the neurotransmitter, norepinephrine, which acts through pancreatic adrenergic receptors. We addressed the effects of blockade of sympathetic neurotransmission on islet morphology and function in mice via expression of tetanus toxin. Silencing of sympathetic activity resulted in defects in islet cyto-architecture and impaired expression of islet cell adhesion markers, similar to observations in sympathectomized mice. Surprisingly, "silenced" animals displayed improved glucose tolerance, enhanced glucose-stimulated insulin secretion, and higher insulin responsiveness, in contrast to findings in sympathectomized mice. These findings are consistent with previous studies describing an acute role for sympathetic activity in inhibiting islet insulin secretion and augmenting glucagon secretion. These results suggest that the presence of sympathetic nerves and neural activity exert similar effects on islet morphology during development but have distinct effects on islet function later in life. 